\title{
Subclinical and Clinical Hypothyroidism in Proteinuric Nephropathies: Amelioration of Proteinuria and Preservation of Renal Function by Thyroid Hormone Replacement Therapy
}

\author{
Saddam A.A. Hassan*, El Metwally L. El Shahawy, Ashraf Talaat Mahmoud, \\ Enas Mamdouh Mohamed Ali, Mohamed E. Ibrahim \\ Internal Medicine Department, Faculty of Medicine, Benha University, Benha, Egypt \\ *Corresponding author: Saddam A.A. Hassan, Email: Saddam.ahmed@fmed.bu.edu.eg, Mobile: 01008950489
}

\begin{abstract}
Background: Emerging evidence shows a bi-directional talk between the kidney and thyroid; yet to date, sparse data exist as to the screening and treatment of hypothyroid states, whether Subclinical Hypothyroidism (SCH) or Clinical Hypothyroidism (CHT), in Chronic Kidney Disease (CKD) patients.

Objectives: The aim of the current study was to examine the association between thyroid hypofunction (whether subclinical or clinical) and proteinuric CKD (low eGFR and/or proteinuria), and to assess the impact of treating thyroid hypofunction on the progression of CKD.

Patients and methods: We conducted a prospective cohort study on 100 participants with baseline proteinuric CKD who were subcategorized according to their thyroid status into three groups: 20 euthyroid, 40 (SCH), 40 (CHT). All subjects were then followed up for 2 years after receiving Thyroid Hormone Replacement Therapy (THRT) according to their thyroid status.

Results: At baseline, we found a highly statistically significant association between SCH/CHT and low eGFR and proteinuria (P-value $<0.001$ for both) in univariate and multivariate analyses. After THRT, we found a statistically significant reduction in CKD progression as evidenced by preservation of mean eGFR and decrease in proteinuria in both groups with $\mathrm{SCH}$ and $\mathrm{CHT}$ ( $\mathrm{P}$-value $<0.001$ and 0.016 respectively) after the first 6 months of treatment that persisted over the remainder of 2 -year follow-up period at a P-value $<0.001$.

Conclusion: The current study demonstrated a strong association between baseline proteinuric CKD and hypothyroid states and showed that THRT significantly halted CKD progression in hypothyroid patients who achieved euthyroid state. Keywords: Chronic Kidney Disease (CKD), Proteinuria, Subclinical Hypothyroidism (SCH), Thyroid Hormone Replacement Therapy (THRT).
\end{abstract}

\section{INTRODUCTION}

Growing evidence from epidemiological and experimental studies has consistently demonstrated the co-existence of CKD and hypothyroid states in an incremental and bi-directional pattern; yet, the mechanistic pathways linking the two disorder, to prove causality, are still evolving ${ }^{(\mathbf{1 - 4})}$.

In CKD, low T3 levels are the most commonly reported thyroid abnormality and $\mathrm{SCH}$ is the most frequent thyroid disorder ${ }^{(5)}$. The uremic milieu in CKD can adversely affect thyroid functions due to altered thyroid hormone synthesis, metabolism, and regulation especially in proteinuric states via: decreased TSH release from hypothalamic-pituitary axis, increased thyroid hormone displacement, decreased $\mathrm{T} 4$ to $\mathrm{T} 3$ peripheral conversion, and increased thyroid-binding proteins' clearance $^{(5)}$.

Hypothyroid states, on the other hand, have been demonstrated in contemporary studies to adversely affect renal functions, the hunt study found that low thyroid function, even within the clinically low normal range, is associated with reduced GFR ${ }^{(4)}$. Hypothyroid-CKD interaction has been contributed to a variety of mechanisms including: preglomerular vasoconstriction, reduced renal perfusion and increased vascular resistance hence decreased GFR, decrease in renal sodium reabsorption, and volume depletion ${ }^{(6-7)}$. Furthermore, hypothyroidism has been shown to cause increased capillary permeability to proteins and cause edema which is reversed by THRT ${ }^{(\boldsymbol{8})}$.

In animal models ${ }^{(\mathbf{9 - 1 1})}$ using isotopic renal functions have shown decreased GFR mainly through hemodynamic changes. Furthermore, exogenous THRT in hypothyroid patients has been demonstrated to increase renal perfusion and improve creatinine clearance ${ }^{(\mathbf{1 2}-15)}$.

In addition, autoimmune conditions provide another common link where hypothyroidism has been reported with multiple glomerulopathies, chief among them is membranous nephropathy ${ }^{(\mathbf{1 6})}$.

The present study aimed at assessing the thyroid status in patients with proteinuric CKD and studying the impact of correction of the hypothyroid states on halting the progression of CKD.

This article is an open access article distributed under the terms and conditions of the Creative Commons Attribution (CC BY-SA) license (http://creativecommons.org/licenses/by/4.0/) 


\section{PATIENT AND METHODS}

Between July 2018 and December 2020, a total of 322 patients from Benha University Hospital clinics were assessed for eligibility, patients were initially screened for CKD, increased urinary Albumin-Creatinine ratio (ACR) and thyroid functions including (TSH, Free T3, Free T4 levels). Among them, 100 patients were eligible for enrollment in the study. CKD was defined as per the 2012 KDIGO guidelines as abnormalities of kidney structure or function, present for $>3$ months, with implications for health. CKD was classified based on cause, GFR category, and albuminuria category (CGA) (17). Subclinical hypothyroidism ( $\mathrm{SCH})$ was defined as an elevation in serum thyroid-stimulating hormone (TSH) concentration (normal range $5-10 \mu \mathrm{IU} / \mathrm{mL}$ ) with a normal serum FT4 concentration, whereas clinical primary hypothyroidism was defined as thyroid-stimulating hormone (TSH) concentrations above the reference range and FT4 concentrations below the reference range ${ }^{(\mathbf{1 8})}$.

The variables that were collected and followed up during the study included: age, sex, body height and weight, body mass index (BMI) calculated as weight divided by height squared, Systolic Blood Pressure (SBP), Diastolic Blood Pressure (DBP), Mean arterial pressure (MAP) and comorbidities. Additionally, laboratory data included: Serum creatinine (SCR), estimated Glomerular Filtration Rate (eGFR) $\left(\mathrm{ml} / \mathrm{min} / 1.73 \mathrm{~m}^{2}\right)$ using the Modification of Diet in Renal Disease Study (MDRD) equation, Urinary AlbuminCreatinine Ratio (ACR), serum cholesterol, serum triglycerides, Fasting Blood Glucose (FBS), Glycated Hemoglobin (HbA1c), and serum albumin.

In all participants, serum free $\mathrm{T} 3$, free $\mathrm{T} 4$, and $\mathrm{TSH}$; thyroid function tests were performed using chemiluminescence immunoassay (Roche Diagnostics, Basel, Switzerland).

One hundred Proteinuric CKD patients were identified: 20 patients were euthyroid, 40 had subclinical hypothyroidism and received $\mathrm{L}$. thyroxine and 40 patients had overt hypothyroidism were also treated with L. thyroxine and all the participants were followed up for 24 months with no dropout. All the variables were measured at baseline then every 6 months to the end of the study. Noteworthy, other lines of treatment for proteinuric CKD were individualized.

\section{Ethical approval:}

The study was performed in accordance with the principles and regulations of the Helsinki's declaration. The study protocol was approved by the Ethical Committee of Benha University on 24/6/2018, with approval number $3089 / 2286$. All the participants gave informed written consents in the Arabic language fully explaining the study and highlighting the potential hazards and benefits of Thyroid Hormone Replacement Therapy (THRT).

\section{Inclusion criteria:}

Age $\geq 18$ years, patients with proteinuric CKD

Exclusion criteria:

History of previous hyperthyroidism, history of THRT before the study, history of autoimmune disease, pregnancy, malignancy, chronic liver disease, heart failure, renal transplant, or ESRD.

\section{Thyroid Hormone Replacement Therapy (THRT):}

Levothyroxine used was (synthetic thyroid hormone, L-Thyroxine, GlaxoSmithKline pharmaceutical $(\boldsymbol{U K})$ with concentration $25,50,100 \mathrm{mcg})$. L-thyroxine was given orally once daily on empty stomach 30 minutes -1 hour before breakfast. The treated patients were initially administered L-thyroxine with the lowest dose necessary to normalize serum TSH level, usually 12.5 to $25 \mu \mathrm{g} / \mathrm{d}$. After the start of thyroid hormone supplement, serum TSH concentration was remeasured for follow-up. If the level of TSH remained above or below the normal reference range, the dose of L-thyroxine was adjusted by 12.5 to $25 \mu \mathrm{g} / \mathrm{d}$ until the patient's thyroid function tests were brought to the normal reference range. In the present study, $90 \%$ of patients have achieved the targets in the first 3 months and $97 \%$ have achieved the targets by the first 6-month time.

\section{Statistical methods}

The clinical data were recorded on a report form. These data were tabulated and analyzed using the computer program SPSS (Statistical Package for the Social Sciences) version 26. Quantitative data were represented as mean+standard deviation (SD) when parametric and were compared by one-way ANOVA test or as median and interquartile range (IQR) when nonparametric and were compared by Kruskal-Wallis test for more than 2 groups or Wilcoxon signed-rank test for paired data. Post-hoc Bonferroni test was used for multiple comparison when comparing more than 2 groups. Qualitative data were represented as frequency and percentage and were compared by $\mathrm{X}^{2}$ test. A multivariate regression analysis was conducted on the dependent and independent variables to adjust for any interaction between thyroid functions and CKD to explore whether thyroid hypofunction was independently associated with CKD. A P value $<0.05$ was considered statistically significant $(*)$ while a $\mathrm{P}$ value $<0.01$ was considered highly significant $(* *)$ in all analyses.

\section{RESULTS}

As shown in table (1), compared to patients with euthyroid state, those with subclinical and overt hypothyroidism had no statistically significant difference regarding age, sex, or blood pressure values. 
Table 1: Comparison between the studied groups according to baseline demographics

\begin{tabular}{|l|l|l|l|l|l|l|l|l|}
\hline \multirow{2}{*}{ Characteristic } & \multicolumn{2}{|l|}{$\begin{array}{l}\text { Euthyroid group } \\
(\mathbf{2 0})\end{array}$} & \multicolumn{2}{l}{$\begin{array}{l}\text { Subclinical } \\
\text { hypothyroidism (40) }\end{array}$} & $\begin{array}{l}\text { Overt hypothyroidism } \\
(\mathbf{4 0})\end{array}$ & $\begin{array}{l}\text { Statistical } \\
\text { test (F) }\end{array}$ & P value \\
\cline { 2 - 11 } & Mean & \pm SD & Mean & \pm SD & Mean & \pm SD & & \\
\hline Age & 47.7 & 9.41 & 45.2 & 11.37 & 44.6 & 7.35 & 0.73 & 0.49 \\
\hline BMI & 23.28 & 2.83 & 24.87 & 2.6 & 24.8 & 2.51 & 2.84 & 0.06 \\
\hline Blood Pressure (BP): & & & & & & & & \\
\hline SBP & 133.25 & 10.29 & 130.0 & 10.13 & 133.0 & 8.83 & 1.23 & 0.30 \\
\hline DBP & 83.75 & 6.26 & 85.8 & 4.27 & 86.5 & 4.21 & 17.0 & 0.11 \\
\hline MAP & 100.3 & 7.03 & 98.8 & 7.49 & 101.8 & 5.88 & 5.59 & 0.15 \\
\hline & No & $\%$ & No & $\%$ & No & $\%$ & Chi square & P value \\
\hline Sex & & & & & & & & \\
Male & 10 & 50.0 & 24 & 60.0 & 16 & 40.0 & 3.2 & 0.20 \\
Female & 10 & 50.0 & 16 & 40.0 & 24 & 60.0 & & \\
\hline
\end{tabular}

(BMI: Body Mass Index, SBP: systolic blood pressure, DBP: diastolic blood pressure, MAP: Mean arterial pressure).

As summarized in table (2), univariate analysis showed that compared to patients with euthyroid state, those with subclinical and overt hypothyroidism had a highly statistically significant difference as regard serum creatinine, eGFR, urinary ACR, serum albumin, total cholesterol, and TG.

Table 2: Comparison between studied groups according to baseline laboratory values

\begin{tabular}{|c|c|c|c|c|c|c|c|c|c|}
\hline \multirow[t]{2}{*}{ Variable } & \multicolumn{2}{|c|}{$\begin{array}{l}\text { Euthyroid group } \\
\text { (20) }\end{array}$} & \multicolumn{2}{|c|}{\begin{tabular}{|l|} 
Subclinical \\
hypothyroidism (40)
\end{tabular}} & \multicolumn{2}{|c|}{$\begin{array}{l}\text { Overt } \\
\text { hypothyroidism (40) }\end{array}$} & \multirow{2}{*}{$\begin{array}{l}\text { Post- } \\
\text { hoc } \\
\text { test }\end{array}$} & \multirow{2}{*}{$\begin{array}{l}\text { (Kruskal- } \\
\text { Wallis) } \\
\text { test }\end{array}$} & \multirow[t]{2}{*}{ P value } \\
\hline & Median & IQR & Median & IQR & Median & IQR & & & \\
\hline Creatinine (mg/d L) & 1.0 & $0.90-1.1$ & 1.2 & $1.1-1.4$ & 1.6 & $1.5-1.8$ & $a b c$ & 50.97 & $<0.001 * *$ \\
\hline $\begin{array}{l}\text { eGFR } \\
\left(\mathrm{min} / \mathrm{ml} / 1.73 \mathrm{~m}^{2}\right)\end{array}$ & 75 & \begin{tabular}{|l|}
$72.3-$ \\
79.8 \\
\end{tabular} & 63.74 & $\begin{array}{l}43.22- \\
78.6\end{array}$ & 56.2 & $\begin{array}{l}45.2- \\
67.57\end{array}$ & $a b$ & 24.92 & $<0.001 * *$ \\
\hline $\begin{array}{l}\text { ACR } \\
(\mathrm{mg} / \mathrm{g})\end{array}$ & 432 & $\begin{array}{l}351.7- \\
537.7\end{array}$ & 991 & $\begin{array}{l}978- \\
1035\end{array}$ & 1110 & $980-1160$ & $a b c$ & 54.77 & $<0.001 * *$ \\
\hline $\begin{array}{l}\text { TSH } \\
(\mathrm{mIU} / \mathrm{L} .)\end{array}$ & 3.6 & $2.63-4.0$ & 8.75 & $7.6-8.9$ & 10.1 & $9.2-11.4$ & $a b c$ & 66.29 & $<0.001 * *$ \\
\hline $\begin{array}{l}\text { FT3 } \\
\text { (ng/dL.) }\end{array}$ & 0.95 & $\begin{array}{l}0.80- \\
1.18 \\
\end{array}$ & 0.91 & $\begin{array}{l}0.90- \\
1.1\end{array}$ & 0.30 & $\begin{array}{l}0.20- \\
0.40\end{array}$ & $\mathrm{bc}$ & 71.92 & $<0.001 * *$ \\
\hline $\begin{array}{l}\text { FT4 } \\
\text { (ng/dL.) }\end{array}$ & 1.1 & $0.80-1.2$ & 1.0 & $0.8-1.2$ & 0.35 & $\begin{array}{l}0.21- \\
0.47\end{array}$ & $\mathrm{bc}$ & 70.24 & $<0.001 * *$ \\
\hline S albumin (g/dL.) & 3.9 & 3.63-4.18 & 3.5 & $3.3-3.7$ & 3.3 & $3.1-3.8$ & $\mathrm{ab}$ & 20.37 & $<0.001 * *$ \\
\hline $\begin{array}{l}\text { Cholesterol } \\
(\mathrm{mg} / \mathrm{dL} .)\end{array}$ & 210 & \begin{tabular}{|l|}
$197.2-$ \\
227.7 \\
\end{tabular} & 198 & $\begin{array}{l}187.0- \\
230\end{array}$ & 247.0 & $242-255$ & $\mathrm{bc}$ & 60.82 & $<0.001 * *$ \\
\hline $\begin{array}{l}\text { STG } \\
(\mathrm{mg} / \mathrm{dL} .)\end{array}$ & 129 & $\begin{array}{l}113.5- \\
150.7 \\
\end{array}$ & 156 & $\begin{array}{l}120- \\
158\end{array}$ & 162.5 & $154-170$ & $a b c$ & 34.90 & $<0.001 * *$ \\
\hline $\begin{array}{l}\text { FBS } \\
(\mathrm{mg} / \mathrm{dL} .)\end{array}$ & 100.0 & \begin{tabular}{|l|}
$89-104$ \\
\end{tabular} & 98.0 & $88-105$ & 95.0 & $90-130$ & & 0.89 & 0.64 \\
\hline $\mathrm{HbA1c}$ & 6.0 & $5.9-6.1$ & 5.95 & $5.8-6.2$ & 6.1 & $5.8-7.0$ & & 2.21 & 0.33 \\
\hline $\begin{array}{l}\text { Uric acid } \\
(\mathrm{mg} / \mathrm{dL} .)\end{array}$ & 5.45 & $\begin{array}{l}4.43- \\
5.98 \\
\end{array}$ & 5.6 & $4.0-6.1$ & 5.5 & $5.0-6.9$ & & 2.84 & 0.24 \\
\hline
\end{tabular}

a: Euthyroid and $\mathrm{SCH}$

b: Euthyroid and CHT

c: SCH and CHT

(eGFR: estimated Glomerular Filtration Rate, ACR: urinary Albumin-Creatinine Ratio, TSH: Thyroid Stimulating Hormone, FT3, FT4: Free Thyroid Hormones, S.TG: Serum Triglycerides, FBS: Fasting Blood Sugar, HbA1c: Glycated Hemoglobin).

Even after adjustment of other baseline variables by multivariate regression analysis of eGFR and proteinuria among euthyroid, $\mathrm{SCH}$, and $\mathrm{CHT}$ groups, (P-for- trend remained $<0.001$ ), showing higher standardized coefficient Beta values for baseline TSH compared to other risk factors for low eGFR/proteinuria in both SCH/CHT, whereas standardized coefficient Beta for FT4 levels was significant only in CHT (Tables 3-6). 
Table 3: Multivariate linear regression of eGFR among SCH groups against other variables at baseline

\begin{tabular}{|c|c|c|c|c|c|}
\hline $\begin{array}{l}\text { eGFR } \\
\left(\mathrm{min} . / \mathrm{ml} / 1.73 \mathrm{~m}^{2}\right)\end{array}$ & $\begin{array}{c}\text { Standardized } \\
\text { coefficient Bets }\end{array}$ & $\mathbf{t}$ & value & \begin{tabular}{|c|}
$\begin{array}{c}\text { Lower limit of } \\
95 \% \text { CI }\end{array}$ \\
\end{tabular} & $\begin{array}{l}\text { Upper limit of } \\
95 \% \text { CI }\end{array}$ \\
\hline TSH (mIU/L.) & 0.871 & 8.070 & $<0.001$ & 0.1 & 0.6 \\
\hline FT4 (ng/dL.) & 0.509 & 7.808 & $<0.001$ & 0.5 & 0.9 \\
\hline $\mathbf{A C R}(\mathrm{mg} / \mathrm{g})$ & 0.678 & 7.960 & $<0.001$ & 0.010 & 0.016 \\
\hline MAP & $-0.558-$ & $-7.227-$ & $<0.001$ & $-1.356-$ & $-0.760-$ \\
\hline DBP & $-0.655-$ & $-7.227-$ & $<0.001$ & $-1.356-$ & $-0.760-$ \\
\hline FBS (mg/dL.) & 0.629 & 7.409 & $<0.001$ & 0.333 & 0.642 \\
\hline HbA1c & 0.429 & $-5.272-$ & $<0.001$ & 0.451 & 1.295 \\
\hline s.TG (mg/dL.) & 0.306 & 3.293 & 0.002 & 0.162 & 0.688 \\
\hline s. Cholesterol (mg/dL.) & 0.306 & $-8.315-$ & $<0.001$ & 1.91 & 2.45 \\
\hline Age & 0.112 & 43.667 & $<0.001$ & 16.12 & 19.22 \\
\hline Sex & 0.019 & 35.435 & 0.002 & $-3.564-$ & -1.263 \\
\hline BMI & 0.123 & 49.239 & $<0.001$ & 14.521 & 17.721 \\
\hline R square & \multicolumn{5}{|c|}{0.81} \\
\hline Adjusted r square & \multicolumn{5}{|c|}{0.77} \\
\hline F test & \multicolumn{5}{|c|}{22.95} \\
\hline$P$ value & \multicolumn{5}{|c|}{$<0.001 * *$} \\
\hline
\end{tabular}

(eGFR: estimated Glomerular Filtration Rate, ACR: urinary Albumin-Creatinine Ratio, TSH: Thyroid Stimulating Hormone, FT3, FT4: Free Thyroid Hormones, S.TG: Serum Triglycerides, FBS: Fasting Blood Sugar, HbA1c: Glycated Hemoglobin)

Table 4: Multivariate linear regression of ACR among SCH groups against other variables at baseline

\begin{tabular}{|c|c|c|c|c|c|}
\hline $\begin{array}{l}\mathbf{A C R} \\
(\mathrm{mg} / \mathrm{g})\end{array}$ & $\begin{array}{l}\text { Standardized } \\
\text { coefficient Beta }\end{array}$ & & P value & $\begin{array}{l}\text { Lower limit } \\
\text { of } 95 \% \mathrm{CI}\end{array}$ & $\begin{array}{l}\text { Upper limit of } \\
95 \% \text { CI }\end{array}$ \\
\hline TSH (mIU/L.) & 1.383 & 554.628 & $<0.001$ & 124.718 & 125.640 \\
\hline FT4 (ng/dL.) & 0.100 & 11.421 & $<0.001$ & 568.841 & 816.585 \\
\hline eGFR $\left(\min . / \mathrm{ml} / 1.73 \mathrm{~m}^{2}\right)$ & 0.978 & 7.960 & $<0.001$ & 0.19 & 0.87 \\
\hline FBS (mg/dL.) & 0.589 & 116.453 & $<0.001$ & 23.558 & 24.399 \\
\hline HbA1c & 0.329 & $-4.203-$ & $<0.001$ & 0.651 & 2.295 \\
\hline DBP & 0.607 & 114.210 & $<0.001$ & 33.931 & 35.166 \\
\hline MAP & $-0.858-$ & $-7.227-$ & $<0.001$ & $-1.356-$ & $-2.760-$ \\
\hline S.TG (mg/dL.) & $-0.775-$ & $-300.04-$ & $<0.001$ & $-56.869-$ & $-56.100-$ \\
\hline S. Cholesterol (mg/dL.) & 0.189 & 35.709 & $<0.001$ & 13.613 & 15.264 \\
\hline Age & 0.012 & 12.667 & $<0.001$ & 11.01 & 13.36 \\
\hline Sex & 0.024 & 14.329 & $<0.001$ & $-2.046-$ & -2.03 \\
\hline BMI & $-0.684-$ & -224.78 & $<0.001$ & $-205.745-$ & $-202.040-$ \\
\hline R square & \multicolumn{5}{|l|}{1.0} \\
\hline Adjusted r square & \multicolumn{5}{|l|}{1.0} \\
\hline F test & \multicolumn{5}{|l|}{127713.7} \\
\hline$P$ value & \multicolumn{5}{|l|}{$<0.001 * *$} \\
\hline
\end{tabular}

(eGFR: estimated Glomerular Filtration Rate, ACR: urinary Albumin-Creatinine Ratio, TSH: Thyroid Stimulating Hormone, FT3, FT4: Free Thyroid Hormones, S.TG: Serum Triglycerides, FBS: Fasting Blood Sugar, HbA1c: Glycated Hemoglobin) 
Table 5: Multivariate linear regression of eGFR among CHT group against other baseline variables

\begin{tabular}{|c|c|c|c|c|c|}
\hline $\begin{array}{l}\text { eGFR } \\
\left(\mathrm{min} . / \mathrm{ml} / 1.73 \mathrm{~m}^{2}\right)\end{array}$ & $\begin{array}{l}\text { Standardized } \\
\text { coefficient } \\
\text { Beta } \\
\end{array}$ & $\mathbf{t}$ & $P$ value & $\begin{array}{l}\text { Lower limit of } \\
95 \% \text { CI }\end{array}$ & $\begin{array}{l}\text { Upper limit of } \\
95 \% \text { CI }\end{array}$ \\
\hline TSH (mIU/L.) & -2.563 & 398.359 & $<0.001$ & 0.89 & 1.45 \\
\hline FT4 (ng/dL.) & $-2.126-$ & $-363.827-$ & $<0.001$ & $-154.895-$ & -153.168 \\
\hline $\operatorname{ACR}(\mathrm{mg} / \mathrm{g})$ & $-.516-$ & $-184.785-$ & $<0.001$ & $-0.006-$ & $-0.006-$ \\
\hline $\begin{array}{l}\text { S. Cholesterol } \\
(\mathrm{mg} / \mathrm{dL} .)\end{array}$ & 1.308 & 334.508 & $<0.001$ & 0.986 & 0.998 \\
\hline FBS (mg/dL.) & $-0.769-$ & $-225.157-$ & $<0.001$ & $-0.906-$ & $-0.889-$ \\
\hline HbA1c & 0.295 & $-45.272-$ & $<0.001$ & 0.96 & 2.952 \\
\hline DBP & 1.301 & 201.420 & $<0.001$ & 5.187 & 5.293 \\
\hline MAP & -1.028 & $-5.709-$ & $<0.001$ & $-1.536-$ & $-3.605-$ \\
\hline ACR (mg/g) & $-1.216-$ & $-184.785-$ & $<0.001$ & $-0.36-$ & $-1.56-$ \\
\hline S.TG (mg/dL.) & 0.249 & 84.878 & $<0.001$ & 0.204 & .214 \\
\hline R square & & & & & 1.0 \\
\hline Adjusted r square & & & & & 1.0 \\
\hline F test & & & & & 83815.1 \\
\hline P value & & & & & $<0.001 * *$ \\
\hline
\end{tabular}

(eGFR: estimated Glomerular Filtration Rate, ACR: urinary Albumin-Creatinine Ratio, TSH: Thyroid Stimulating Hormone, FT3, FT4: Free Thyroid Hormones, S.TG: Serum Triglycerides, FBS: Fasting Blood Sugar, HbA1c: Glycated Hemoglobin)

Table 6: Multivariate linear regression of ACR among CHT group against other baseline variables

\begin{tabular}{|c|c|c|c|c|c|}
\hline $\begin{array}{l}\mathbf{A C R} \\
(\mathrm{mg} / \mathrm{g})\end{array}$ & $\begin{array}{l}\text { Standardized } \\
\text { coefficient } \\
\text { Beta }\end{array}$ & & $P$ value & $\begin{array}{l}\text { Lower limit } \\
\text { of } 95 \% \mathrm{CI}\end{array}$ & $\begin{array}{l}\text { Upper limit of } \\
95 \% \text { CI }\end{array}$ \\
\hline TSH (mIU/L.) & 4.158 & 162.265 & $<0.001$ & 323.061 & 331.285 \\
\hline FT4 (ng/dL.) & $-2.662-$ & $-286.690-$ & $<0.001$ & $-0.518-$ & $-0.919-$ \\
\hline eGFR $\left(\mathrm{min} . / \mathrm{ml} / 1.73 \mathrm{~m}^{2}\right)$ & $-2.099-$ & $-321.969-$ & $<0.001$ & -176.090 & $-173.873-$ \\
\hline FBS $(\mathrm{mg} / \mathrm{dL})$. & $-0.891-$ & -521.549 & $<0.001$ & $-1.356-$ & $-2.765-$ \\
\hline HbA1c & -0.765 & $-345.954-$ & $<0.001$ & $-2.654-$ & $-2.576-$ \\
\hline DBP & $-1.09-$ & $-230.085-$ & $<0.001$ & $-1.26-$ & $-2.18-$ \\
\hline MAP & 1.109 & 196.078 & $<0.001$ & 602.474 & 615.140 \\
\hline S. cholesterol (mg/dL.) & 0.410 & 297.042 & $<0.001$ & 520.325 & 527.519 \\
\hline S. TG (mg/dL.) & 0.158 & 162.265 & $<0.001$ & 323.061 & 331.285 \\
\hline Age & $-0.406-$ & $-463.053-$ & $<0.001$ & $-224.956-$ & $-222.983-$ \\
\hline BMI & $-0.141-$ & $-52.539-$ & $<0.001$ & $-588.653-$ & $-544.658-$ \\
\hline R square & & & & & 1.0 \\
\hline Adjusted r square & & & & & 1.0 \\
\hline F test & & & & & 1435091.9 \\
\hline P value & & & & & $<0.001 * *$ \\
\hline
\end{tabular}

(eGFR: estimated Glomerular Filtration Rate, ACR: urinary Albumin-Creatinine Ratio, TSH: Thyroid Stimulating Hormone, FT3, FT4: Free Thyroid Hormones, S.TG: Serum Triglycerides, FBS: Fasting Blood Sugar, HbA1c: Glycated Hemoglobin).

The impact of THRT on eGFR (at 6, 12, 18, 24 months) is summarized in table (7) showing that in patients with subclinical hypothyroidism and clinical hypothyroidism, there was a highly

statistically significant improvement of eGFR from baseline values along all the follow- up period (all $\mathrm{p}$ values $<0.001$ ); nevertheless, the most clinically significant improvement was noticed early on after the first 6 months, then the trend continued to improve afterward albeit steeply. 
Table 7: Differences of estimated GFR from baseline and along follow up periods after thyroid replacement therapy among subclinical and overt hypothyroidism

\begin{tabular}{|c|c|c|c|c|}
\hline \multirow[t]{2}{*}{ Subclinical hypothyroidism (40) } & \multicolumn{2}{|c|}{ eGFR $\left(\mathrm{min} . / \mathrm{ml} / 1.73 \mathrm{~m}^{2}\right)$} & \multicolumn{2}{|c|}{ Base line and } \\
\hline & Median & IQR & $\begin{array}{l}\text { Wilcoxon } \\
\text { signed-rank } \\
\text { test }\end{array}$ & $\mathbf{P}$ \\
\hline Base line & 63.74 & $43.22-78.68$ & & \\
\hline After 6 months follow up & 71.39 & $51.63-81.94$ & 5.24 & $<0.001 * *$ \\
\hline After 12 months follow up & 71.39 & 51.4- 87.23 & 5.52 & $<0.001 * *$ \\
\hline After 18 months follow up & 71.80 & $51.83-87.33$ & 5.54 & $<0.001 * *$ \\
\hline After 24 months follow up & 72.0 & $51.59-87.40$ & 5.22 & $<0.001 * *$ \\
\hline \multirow[t]{2}{*}{ Overt hypothyroidism (40) } & \multicolumn{2}{|c|}{$\begin{array}{l}\text { eGFR } \\
\left(\mathrm{min} . / \mathrm{ml} / 1.73 \mathrm{~m}^{2}\right)\end{array}$} & \multicolumn{2}{|c|}{ Base line and } \\
\hline & Median & IQR & $\begin{array}{l}\text { Wilcoxon } \\
\text { signed-rank } \\
\text { test } \\
\end{array}$ & $\mathbf{P}$ \\
\hline Base line & 56.20 & $45.2-67.57$ & & \\
\hline After 6 months follow up & 60.86 & $49.57-71.16$ & 5.52 & $<0.001 * *$ \\
\hline After 12 months follow up & 60.62 & 49.39- 71.41 & 5.22 & $<0.001 * *$ \\
\hline After 18 months follow up & 61.09 & $49.80-71.60$ & 5.24 & $<0.001 * *$ \\
\hline After 24 months follow up & 61.22 & $50.88-71.99$ & 5.12 & $<0.001 * *$ \\
\hline
\end{tabular}

e GFR: estimated Glomerular Filtration Rate, IQR: Inter Quartile Range, MWZ test: Mann-Wilcoxon-Whitney test

In table (8), the impact of THRT on urinary ACR comparing baseline values with values at 6, 12, 18, 24 months is listed showing a similar trend for proteinuria reduction to that of improved eGFR along the time, whereas in the subclinical hypothyroid and clinical hypothyroid groups we found a highly statistically significant reduction of ACR from a baseline along follow up period, that was again most clinically significant after the first 6 months, then continued afterwards albeit at a steeper rate of proteinuria reduction.

Table 8: Differences in urinary Albumin-Creatinine Ratios (ACR) from baseline and along follow up periods after thyroid replacement therapy among subclinical and overt hypothyroidism

\begin{tabular}{|l|l|l|l|l|}
\hline \multirow{2}{*}{$\begin{array}{l}\text { Subclinical hypothyroidism } \\
\text { (40) }\end{array}$} & \multicolumn{2}{|l|}{ ACR $(\mathbf{m g} / \mathbf{g})$} & \multicolumn{2}{l|}{ Base line and } \\
\cline { 2 - 5 } & Median & IQR & $\begin{array}{l}\text { Wilcoxon } \\
\text { signed-rank test }\end{array}$ & P \\
\hline Base line & 991.0 & $978.0-1035.0$ & & \\
\hline After 6m follow up & 596.5 & $554.0-670.0$ & 5.52 & $<0.001^{* *}$ \\
\hline After 12m follow up & 575.0 & $534.0-660.0$ & 5.52 & $<0.001^{* *}$ \\
\hline After 18m follow up & 565.0 & $522.0-644.0$ & 5.12 & $<0.001^{* *}$ \\
\hline After 24m follow up & 556.0 & $490.0-630.0$ & 5.56 & $<0.001^{* *}$ \\
\hline Overt hypothyroidism (40) & ACR (mg/g) & \multicolumn{2}{|l|}{} \\
\cline { 2 - 5 } & Median & IQR & $\begin{array}{l}\text { Wilcoxon } \\
\text { signed-rank test }\end{array}$ & P \\
\hline Base line & \multicolumn{4}{|l}{} \\
\hline After 6m follow up & 1110.5 & $980.0-1160.0$ & 5.52 & $<0.001^{* *}$ \\
\hline After 12m follow up & 822.0 & $756.0-944.0$ & 5.52 & $<0.001^{* *}$ \\
\hline After 18m follow up & 795.0 & $745.0-867.0$ & $5.001^{* *}$ \\
\hline After 24m follow up & 742.0 & $699.0-845.0$ & 5.5 & $<0.001^{* *}$ \\
\hline
\end{tabular}

ACR: urinary Albumin-Creatinine Ratio, IQR: Inter Quartile Range, MW test: Mann-Wilcoxon-Whitney test 


\section{DISCUSSION}

The findings of the current study demonstrating an association between CKD and SCH/CHT come in agreement with previous observational cross-sectional studies ${ }^{(\mathbf{1 - 4})}$. Nevertheless, a significant limitation of the previous studies showing such association comes from their inherently limited ability to establish a mechanistic link for temporal causality. The present study demonstrated a statistically significant association between baseline proteinuric CKD and SCH/CHT. We conducted a univariate analysis and a multivariate analysis to adjust for the interaction of the baseline risk factors for CKD (Low eGFR/ Proteinuria), in both univariate and multivariate models, $\mathrm{SCH} / \mathrm{CHT}$ were associated with CKD.

Long considered a physiological adaptation in CKD, evolving evidence on hypothyroid states challenges this notion suggesting that low thyroid hormone levels, left untreated, might be a risk factor for both incident CKD and CKD progression (19-21). Therefore, we examined the impact of correcting thyroid hypofunction to an euthyroid state by THRT on the progression of baseline CKD, we found that THRT in both $\mathrm{SCH}$ and $\mathrm{CHT}$ was associated with a statistically significant improvement in eGFR levels and decrease in proteinuria levels along the follow-up, the beneficial effect of THRT started and was most clinically significant after the first 6 months on treatment and continued all over the 2-year follow-up period. Several studies have shown a beneficial effect on reversing hypothyroid state on halting the progression of CKD ${ }^{\text {(11- }}$ 14). In CKD patients with $\mathrm{SCH}$, unresolved $\mathrm{SCH}$ was found as an independent predictor of CKD progression, interestingly enough, in this same study, spontaneous resolution of SCH even without treatment has led to less decline in eGFR ${ }^{(19)}$. Our current study findings confirm and strengthen the favorable kidney outcomes conveyed by exogenous THRT in both SCH and CHT groups with baseline proteinuric CKD.

Whilst the consensus in Endocrinology Society dictates starting THRT for patients with CHT albeit in small increments in elderly patients, in $\mathrm{SCH}$, indications for starting THRT are rather individualized based on the risk for progression to CHT if TSH levels $>10 \mu \mathrm{IU} / \mathrm{mL}$ or in the presence of thyroid peroxidase antibodies (TPO) or symptoms or comorbidities. Yet, there is no explicit recommendations in endocrinology, nor in nephrology guidelines regarding the screening or treatment of hypothyroid states in CKD despite the growing evidence suggesting a causal interplay between the two entities even independent of conventional risk factors ${ }^{(21)}$.

Proteinuric $\mathrm{CKD}$ in patients with $\mathrm{SCH}$ even poses a more important clinical question regarding the benefit of starting HTRT merely for preservation of renal functions in the absence of other traditional indications. Shin $\boldsymbol{e t} \boldsymbol{a l}{ }^{(22-23)}$, showed that patients with subclinical hypothyroidism who received THRT had a lower risk of CKD progression compared to their counterparts with $\mathrm{SCH}$ who did not receive treatment even after adjusting for other conventional risk factors for CKD progression. Our current study showed a similar effect of THRT for halting CKD progression. Taken together, the question that springs to mind is whether the presence of CKD alone (reduced eGFR/ Proteinuria) could be a novel indication for treatment of subclinical hypothyroidism? Obviously, further larger and longer studies are needed to explore this potential.

In addition, the cut offs used in our present study as well as previous studies to define hypothyroid status are derived from studies conducted on general population ${ }^{(1-4)}$. CKD patients are well-known to have distinct features that distinguish them from the normal population. Hence, if we are to accurately distinguish true thyroid disorders from non-thyroidal illness in CKD patients, further research is warranted to identify more sensitive and specific methods to classify thyroid disorders in CKD population. Furthermore, the dosing and targets of THRT in CKD patients need to be further explored in future studies.

Taken together, our results demonstrate the benefit and call for the screening and treatment of thyroid hypofunction states in patients with proteinuric $\mathrm{CKD}$ as a potential untraditional risk factor for CKD progression.

\section{LIMITATIONS}

It is fair to acknowledge that our study has some salient limitations including:

1- The average age in our cohort was around late forties, relatively a young population, hence no adverse events regarding ischemia, arrhythmias, or bone mineral disorders were reported with THRT; nevertheless, this should be kept in mind when deciding on elderly patients with $\mathrm{SCH}$ who are more liable for side effects of THRT.

2- A small number of patients with a short follow-up period, therefore, larger studies with longer follow-up periods are needed to further explore our findings.

3 - In the present study, we used MDRD equation as a surrogate for eGFR. Notwithstanding their imperfections and limitations of performance of $\mathrm{e}$ GFR equations, our findings are consistent with the results of studies that have used radio-isotope imaging for measuring GFR in hypothyroid states and their treatment ${ }^{(\mathbf{1 2}-13)}$.

4- Screening for thyroid peroxidase (TPO) was not routinely ordered in our cohort, however, the HUNT 
study did not find a difference between those with or without TPO in terms of CKD ${ }^{(4)}$.

\section{CONCLUSION}

Our findings strengthen and support the previous reports on the reciprocal relation between CKD and hypothyroid states; and demonstrate that THRT can be safely advocated to preserve renal functions in proteinuric CKD patients with $\mathrm{SCH} / \mathrm{CHT}$ suggesting a potential for halting CKD progression after THRT in both $\mathrm{SCH} / \mathrm{CHT}$ that warrants to be confirmed in future studies.

\section{REFERENCES}

1. Chang Y, Chang C, Yeh Y et al. (2018): Subclinical and overt hypothyroidism is associated with reduced glomerular filtration rate and proteinuria: a large cross-sectional population study. Kidney International. Sci Rep., 8(1):2031.

2. Chonchol M, Lippi G, Salvagno G, Zoppini G, Muggeo M, Targher G (2008): Prevalence of subclinical hypothyroidism in patients with chronic kidney disease. Clin J Am Soc Nephrol., 3: 1296-300.

3. Lo J, Chertow G, Go A, Hsu C (2005): Increased prevalence of subclinical and clinical hypothyroidism in persons with chronic kidney disease. Kidney Int., 67:104752.

4. Bjørn O , Trine B, Lars V (2011): Association of thyroid function with estimated glomerular filtration rate in a population-based study: the HUNT study. Eur $J$ Endocrinol., 164: 101-5.

5. Iglesias P, Díez J (2009): Thyroid dysfunction and kidney disease. Eur J Endocrinol., 160: 503-515

6. Toft A, Boon N (2000): Thyroid disease and the heart. Heart, 84: 455-60.

7. Klein I, Danzi S (2007): Thyroid disease and the heart. Circulation, 116: 1725-35.

8. Wheatley $\mathbf{T}$,Edwards $\mathrm{O}$ (1983): Mild hypothyroidism and oedema: evidence for increased capillary permeability to protein. Clin Endocrinol (Oxf), 18: 627-35. 9. Falk S, Buric V, Hammond W, Conger J (1991): Serial glomerular and tubular dynamics in thyroidectomized rats with remnant kidneys. Am J Kidney Dis., 17: 218-27.

10. Bradley S, Stéphan F, Coelho J, Réville P (1974): The thyroid and the kidney. Kidney Int., 6: 346-65.
11. Karanikas G, Schütz M, Szabo M et al. (2004): Isotopic renal function studies in severe hypothyroidism and after thyroid hormone replacement therapy. Am J Nephrol., 24: 41-5.

12. Capasso G, De Tommaso G, Pica A et al. (1999): Effects of thyroid hormones on heart and kidney functions. Miner Electrolyte Metab., 25: 56-64.

13. R. Hajji, F. Derbali, K. Mnafgui et al. (2014): A transient proteinuria: An unusual complication of hypothyroidism. American Journal of Medical Case Reports, 2(11): 237-239.

14. Mooraki A, Broumand B, Neekdoost F, Amirmokri P ,Bastani B (2003): Reversible acute renal failure associated with hypothyroidism: report of four cases with a brief review of literature. Nephrology (Carlton), 8: 57-60.

15. Andrew C ,Joanne E (2008): Renal impairment resulting from hypothyroidism. NDT Plus, 1:440-441.

16. Santoro $\mathrm{D}$, Vadalà $\mathrm{C}$, Siligato $\mathrm{R}$ et al. (2017): Autoimmune Thyroiditis and autoimmune glomerulonephritis. Front Endocrinol (Lausanne), 8:119.

17. Kidney Disease: Improving Global Outcomes (KDIGO) CKD Work Group (2012): Clinical Practice Guideline for the Evaluation and Management of Chronic Kidney Disease. Kidney inter., 3: 1-150.

18. Peeters R (2017): Subclinical hypothyroidism. N Engl J Med., 376(26):2556-2565.

19. Eun O, Ihn S, Yoo A, Sang J et al. (2014): Unresolved subclinical hypothyroidism is independently associated with progression of chronic kidney disease. International Journal of Medical Sciences, 11(1):52-59.

20. Connie M (2016): The interaction between thyroid and kidney disease: An overview of the evidence. Curr Opin Endocrinol Diabetes Obes., 23(5): 407-415.

21. Xiaodong W, Xinchao Z, Xiaolei H (2020): Association of subclinical thyroid dysfunction with chronic kidney disease: A systematic review and meta-analysis, Endocrine Research, 45:1, 41-49.

22. Shin D et al. (2013): Thyroid hormone replacement therapy attenuates the decline of renal function in chronic kidney disease patients with subclinical hypothyroidism. Thyroid, 23: 654-661.

23. Shin D et al. (2012): Preservation of renal function by thyroid hormone replacement therapy in chronic kidney disease patients with subclinical hypothyroidism. J Clin Endocrinol Metab., 97: 2732-40. 